\title{
X-RAY CT Reconstruction by using Spatially Non Homogeneous ICD Optimization
}

\author{
U. Satheeshwaran, N. Sreekanth, J.Surendiran
}

\begin{abstract}
Recent applications of conventional iterative coordinate descent (ICD) algorithms to multislice helical CT reconstructions have shown that conventional ICD can greatly improve image quality by increasing resolution as well as reducing noise and some artifacts. However, high computational cost and long reconstruction times remain as a barrier to the use of conventional algorithm in the practical applications. Among the various iterative methods that have been studied for conventional, ICD has been found to have relatively low overall computational requirements due to its fast convergence. This paper presents a fast model-based

iterative reconstruction algorithm using spatially nonhomogeneous ICD (NH-ICD) optimization. The NH-ICD algorithm speeds up convergence by focusing computation where it is most needed. The NH-ICD algorithm has a mechanism that adaptively selects voxels for update. First, a voxel selection criterion VSC determines the voxels in greatest need of update. Then a voxel selection algorithm VSA selects the order of successive voxel updates based upon the need for repeated updates of some locations, while retaining characteristics for global convergence. In order to speed up each voxel update, we also propose a fast 3-D optimization algorithm that uses a quadratic substitute function to upper bound the local 3-D objective function, so that a closed form solution can be obtained rather than using a computationally expensive line search algorithm. The experimental results show that the proposed method accelerates the reconstructions by roughly a factor of three on average for typical 3-D multislice geometries.
\end{abstract}

Index Terms - Computed tomography, coordinate descent, iterative algorithm, conventional algorithm

\section{INTRODUCTION}

In an effort to speed up the iterative reconstruction, various hardware platforms are being considered [1], [2], and a variety of iterative methods, such as variations of expectation maximization (EM) [3], conjugate gradients (CG) [4], ordered subsets (OS) [5], and iterative coordinate descent (ICD) [6], is typically used to minimize the objective function. Each iteration of these methods can be computationally expensive since it typically requires at

Revised Manuscript Received on 14 September, 2019.

J.Surendiran, Professor, Dept. of ECE, HKBKCE, Bangalore. E-mail: surenjaya1981@gmail.com

Dr.U.Satheeshwaran, Professor, Dept. of ECE, Malla Reddy Engineering College for Women(Autonomous), Secunderabad-TS

Dr. N. Sreekanth, Professor, Dept. of ECE, Malla Reddy Engineering College for Women(Autonomous), Secunderabad-TS E-mail: nsreeku@gmail.com least one pass through a large volume of CT data, and the number of required iterations depends upon both the desired image quality and the convergence speed of the particular iterative algorithm. Among various iterative methods that have been applied to conventional, ICD, and similar sequential updating techniques such as OS-ICD [7], [8] and groupcoordinate ascent [9], [10], have been found to have relatively low overall computational requirements. The convergence behavior of the ICD algorithm has been studied in the literature [11], [12]. In fact, among the optimization algorithms compared in [13], the ICD algorithm was found to have a relatively fast convergence behavior when it is initialized with the Filter Back Projection (FBP) reconstruction, which usually provides a good estimate of the low spatial frequency content of the image that tends to converge more slowly with the ICD algorithm. It should be noted that ICD tends to have less regular memory access than gradient based optimization methods. The ICD algorithm works by decomposing the $\mathrm{N}$-dimensional optimization problem into a sequence of greedy 1-D voxel updates. A full iteration of the conventional ICD algorithm then updates all the voxels in the image volume once and once only. In addition to the fast convergence speed, ICD has also been found to have a number of useful properties in model-based iterative reconstruction. First, the ICD algorithm can easily incorporate positivity constraints and non-Gaussian prior distributions. Second, the ICD algorithm naturally allows for updates that vary nonuniformly across the reconstructed image.

Proposed system is nonhomogeneous ICD (NH-ICD) algorithm [14] that can substantially accelerate convergence relative to the conventional ICD algorithm for tomographic reconstruction. The NH-ICD algorithm takes advantage of the flexibility of ICD by selectively updating voxels that can benefit most from updates. Typically, the errors between the FBP initialization of ICD and the converged reconstruction are not uniformly distributed across the image. In fact, these initial errors tend to be primarily distributed around edges and other localized regions. Therefore, the convergence speed of ICD can be improved by focusing computational resources on these important locations. In order to select the order of voxel updates, we formulate a voxel selection criterion (VSC) to determine the voxels in greatest need of update. We also develop a voxel selection algorithm (VSA) that balances the need for repeated updates of some voxels with the need for more uniform updating of all voxels to guarantee global convergence. It also proposes a fast algorithm for

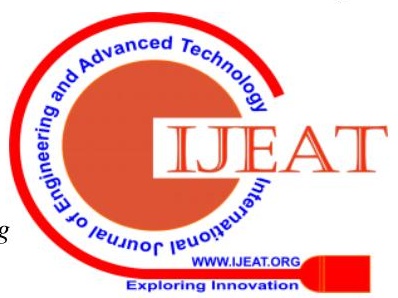

Blue Eyes Intelligence Engineering \& Sciences Publication 


\section{X-RAY CT Reconstruction by using Spatially Non Homogeneous ICD Optimization}

approximately solving the 1-D optimization problem of each voxel update [15] in order to speed up the ICD algorithm. The fast 1-D update algorithm is based on the functional substitution (FS) approach [9], [16], [17], which replaces the objective function with a simplified

substitute function. By carefully deriving the substitute function, the FS approach reduces computation while also guaranteeing monotone convergence of the objective function.

Here, the performances of the proposed algorithms using several clinical data sets which cover a variety of anatomical locations are examined. The experimental results show that the proposed algorithms reduce the computation time required to achieve desired image quality by approximately a factor of three on average as compared to the conventional ICD algorithm. The content is organized as follows. Section II provides a review of the conventional ICD algorithm for 3-D iterative reconstruction. Section III presents the spatially nonhomogeneous ICD algorithm. Finally, in Section IV the experimental results on clinical data cases to quantify the improvement in computation speed.

\section{CONVENTIONAL ICD ALGORITHM FOR 3-D RECONSTRUCTION}

\section{A. Statistical Model and Objective Function}

The conventional ICD algorithm for reconstruction of 3D volumes from data obtained using a multislice CT system was introduced. To define the coordinate system for the reconstructed image volume, let, $\mathrm{x}, \mathrm{y}$, and $\mathrm{z}$ and denote the basis vectors of a right-handed coordinate system. The origin of the coordinate system is placed at the center of the rotation, also known as the "isocenter" of the scanner. The distance driven (DD) forward model is choosen mainly because it is relatively fast to compute, and it has been shown to produce images free of visible aliasing artifacts introduced by the forward model [19]. To forward project one voxel using the DD model, we first "flatten" the voxel to a rectangle shape. Then we compute the projection of the four boundaries of this flattened voxel onto the detector array. The projection is approximated as a rectangle shape and can be specified by its width, length and the center location in the detector coordinate system. Consequently, the location and the size of the rectangular footprint can be separately computed in the $(\mathrm{x}, \mathrm{y})$ plane and along the $\mathrm{z}$ axis. Therefore, the forward model can be calculated separately in the $(x, y)$ plane and the $\mathrm{z}$ plane. Later we will discuss how to use the separability to efficiently calculate the forward projection of a line of voxels that are parallel to the $\mathrm{z}$ axis.

\section{B. ICD Algorithm}

One full iteration of the ICD algorithm works by updating voxels in sequence, until every voxel has been updated exactly once. Each voxel is updated so as to globally minimize the total objective function while fixing the remaining voxels. The FBP reconstruction typically provides a good initial condition for the ICD algorithm. This is because the FBP generally provides an

accurate estimate of the low frequency components of the reconstruction.

Higher frequency edge and texture details are generally not as accurate in the FBP images, but the ICD algorithm is known to have rapid convergence at high frequencies [6]. Each voxel update of ICD requires the computation of the 1-D minimization. This update can be done using half-interval search, which is simple and robust, but relatively slow because it requires multiple steps to reach the desired precision of solution. Moreover, the number of steps required for a given precision may vary between voxel updates. In each iteration of the conventional ICD algorithm, each voxel is updated once and only once. However, the order of voxel updates may vary with each iteration. We follow two rules in selecting the order of voxel updates. First, entire lines of voxels along the $\mathrm{z}$ axis are updated in sequence. The voxels in the voxel-line share the same geometry calculation in the $(\mathrm{x}, \mathrm{y})$ plane. Second, we update the voxel-lines in a random order, so that each voxel-line is updated once per iteration, and the order of selection is randomized with a uniform distribution [18]. The figure.1 (Fig.1) explains that from the original image noise and blur are added and it is removed by this algorithm. The PSNR value is given after each iteration.

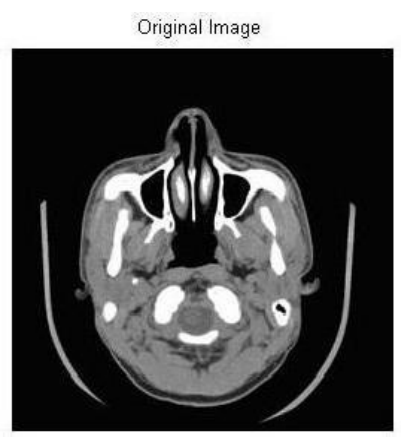

(a)

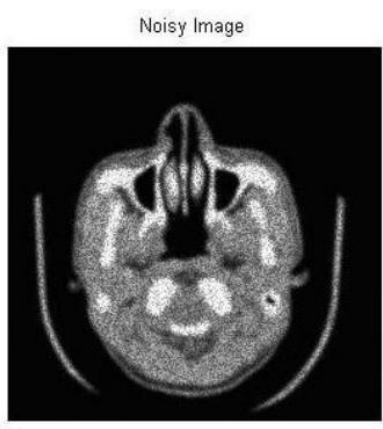

(c)

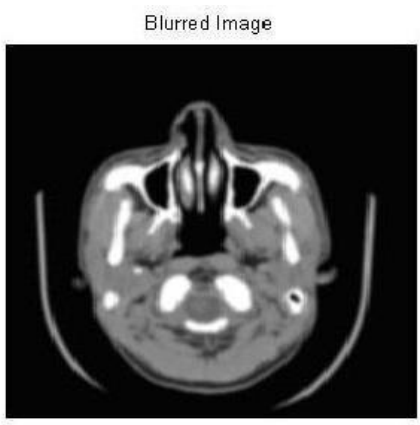

(b)

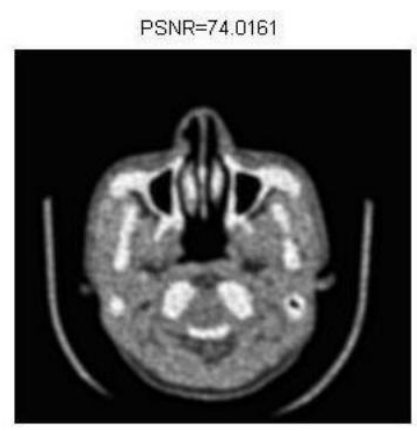

(d) 


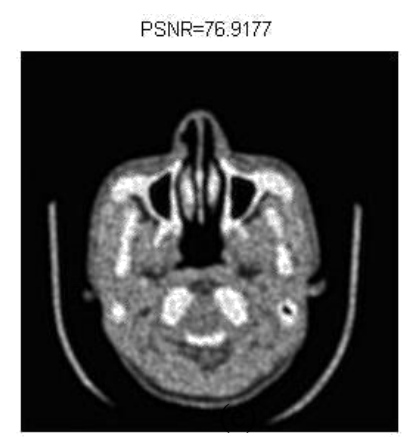

Fig.1. (a) input (original) image (b) blur image (c) noise image (d) Denoisy image (first iteration) (e) Denoisy image (second iteration)

\section{SPATIALLY NONHOMOGENEOUS ICD (NH-ICD) ALGORITHM}

The basic idea behind the spatially NH-ICD algorithm is updating some voxel-lines more frequently than others. The NH-ICD algorithm is motivated by the fact that the convergence error, which we define as the error between the current value and the fully converged value of a voxel, is not uniformly distributed across a reconstructed image. In fact, the convergence error tends to be distributed primarily near edges. However, the conventional ICD algorithm does not exploit this nonuniform distribution of error because each voxel must be updated exactly once per iteration. Therefore, the NH-ICD algorithm was proposed to improve the convergence speed of ICD by focusing computational resources on the voxel-lines which can benefit most from updates.

In order to implement the NH-ICD algorithm, one must determine an ordering of the voxel updates which yields fast convergence to the maximum a posteriori (MAP) estimate. Notice that the greedy selection method actually has slower convergence than conventional ICD. This is because fast convergence also requires that some voxels with lower MSE be updated, but these updates can be less frequent. Moreover, even if it worked well, this greedy selection method cannot be practically implemented because it depends upon the knowledge of the converged MAP reconstruction to compute the MSE of each voxel-line. With this example in mind, nonhomogeneous ICD algorithm will be based on two concepts. First, we will compute a VSC for each voxel-line. The VSC will be used to determine which voxel-lines are in greatest need of update. Second, at a higher level, VSA is also needed. The VSA will be designed to balance the need for repeated dates of some voxel lines with the need for more uniform updating of all voxels lines.

\section{A. VSC (Voxel Select Criterion)}

The VSC was choosen to be related to the absolute sum of the update magnitudes along a voxel-line at its last visit. Intuitively, if a voxel-line had large updates, then it is likely that the voxels are far from their converged values, and could benefit from more frequent selection. The VSC is not available until after the first full ICD update. In Section III-C, the interleaved NH-ICD algorithm was introduced, which is designed to overcome this limitation. It is found empirically that adjacent voxels have strong correlation in their MSE. Therefore, the low-pass filter is used to encourage updates of local neighborhoods and to reduce the variation in the VSC.

Fig.1. (a) shows that input image which is scanned and Fig.2. (b) shows the voxel selected image.

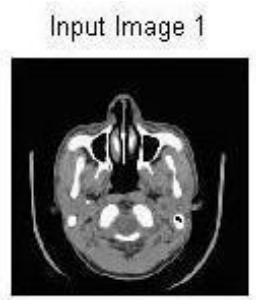

Voxels 1

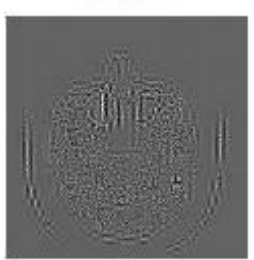

\section{B. VSA (Voxel Select Algorithm)}

Therefore, an effective VSA must balance the need for repeated updating of some voxels with the need for improvement of the remainder of the image. In order to achieve this goal, two subprocedures in the VSA were incorporated. The nonhomogeneous subprocedure selects voxel-lines that have large VSC values, and updates them frequently. Alternatively, the homogeneous subprocedures update all voxel-lines uniformly. By alternating between these two subprocedures, the convergence of the voxels with large VSC values is accelerated while ensuring that all voxel-lines and their VSC values are updated. The algorithm starts with a homogeneous subprocedure, in which each voxel line is updated exactly once, in a randomized order.

This first homogeneous subprocedure ensures that the values of the update magnitude map once the first homogeneous subprocedure is completed, the NH-ICD algorithm iterates between a nonhomogeneous subprocedure and a homogeneous subprocedure, and these two steps are repeated until the desired level of convergence is achieved. When updating a voxel-line, we sometimes ignore zero-valued voxels in a process we call "zero-skipping." So, that typically many voxels in air converge were found to a value

of zero due to the positivity constraint, and can be skipped with little effect on the reconstructed image. Thus, if a voxel and its neighbors all have value zero, the ICD update of that voxel was skipped. A voxel-line can be updated at most once in one subiteration, but if it produces a large update magnitude, it may be selected in subsequent subiterations. Therefore, a voxel-line can be updated as many as $\mathrm{K}$ times during a single application of the non homogeneous sub procedure.

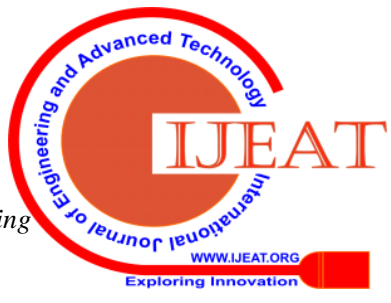




\section{X-RAY CT Reconstruction by using Spatially Non Homogeneous ICD Optimization}

\section{Interleaved NH-ICD}

Interleaved NH-ICD is intended to allow nonhomogeneous subprocedures before the completion of the first full homogeneous update, to exploit as early as possible the knowledge of the locations where updates are most needed. To do this, a densely interleaved set of voxel-lines was updated, so that a value for VSC can be computed at all voxel locations. This allows the first nonhomogeneous subprocedure to be run after a fraction of iteration. The interleaved NH-ICD algorithm starts by performing a homogeneous update of only the voxel-lines. The partial nonhomogeneous subprocedure then updates the voxel-lines with the largest VSC. The number of voxel updates performed in this partial nonhomogeneous subprocedure is proportional to the number in the previous partial homogeneous procedure. This process is repeated four times until each subset has been updated once, after which the NH-ICD algorithm alternates between full homogeneous and nonhomogeneous subprocedures until convergence is achieved. In the partial nonhomogeneous subprocedure, the VSC for each voxel-line using partially initialized update magnitude maps were computed. Therefore, the updates in the partial nonhomogeneous subprocedure are not limited to the subset. Since the voxel-lines are uniformly sampled in the partial homogeneous subprocedures, the filtering step can be viewed as a simple interpolation which fills in the values in the VSC for the voxel-lines that have not yet been updated.

\section{EXPERIMENTAL RESULTS}

The algorithm was parallelized so that each core was responsible for updating a sequence of slices along the axis. All the cores simultaneously work on the same voxel-line. Once a voxel-line is selected, voxel updates onto each core were distributed. Moreover, the voxels being updated were guaranteed in parallel are far apart so that they do not share any sinogram data and can be updated independently. The functional substitution method performs only one update for each voxel. The computation time is measured by averaging all the voxel update times from 10 iterations of the conventional ICD algorithm. The results show that the functional substitution algorithm on average reduces the computation time of the 1-D optimization by $85 \%$. Consequently, the total computation time per voxel update is reduced by approximately $20 \%$ on this computer. Since the two algorithms do not reach same numerical solution at each voxel update, we would also compare the convergence speed. In order to compare the speed of convergence for each of these methods, we need measures of both convergence and computation. Two measures of convergence were employed. The first measure is the value of the MAP cost function being minimized. The second measure is the RMSE difference between the current image and its converged value after 50 iterations of the conventional ICD algorithm. Two measures of computation were also used. The first measure, called equivalent iteration or "equit," is based on the total number of voxel updates and is defined as,

Number of voxel updates

equit $=\frac{\text { Total number of voxels in the field of view (FOV) }}{\text { Tow }}$
By this definition, one conventional ICD iteration requires one equit of computation. The subprocedures in NH-ICD generally require less than one equit due to zero-skipping. Also, one equit of NH-ICD may update some voxels multiple times whereas other voxels are not visited. The second measure of computation is the normalized wall clock time, which is computed as the actual wall clock time divided by the computation time required for a single full iteration.

\section{CONCLUSION}

Spatially nonhomogeneous ICD algorithm with 1- D optimization was presented. The method works by focusing computation to the most important areas of the reconstruction. The experiments on a variety of clinical data sets show that the proposed algorithm accelerates the reconstruction by a factor of approximately three on average. This improved convergence speed may be used to either reduce computation for a fixed level or improve quality for applications with fixed computational resources.

\section{REFERENCES}

1. K. Mueller and F. Xu, "Practical considerations for GPU-accelerated CT," in PROC. 3rd IEEE Int. Symp. Biomed.

2. Imag.: Nano to Macro, Apr. 2006, pp. 1184-1187.

3. M. Kachelrieß, M. Knaup, and O. Bockenbach, "Hyperfast parallelbeam and cone-beam backprojection using the cell general purpose hardware,” Med. Phys., vol. 34, no. 4, pp. 1474-1486, 2007.

4. L. Shepp and Y. Vardi, "Maximum likelihood reconstruction for emission tomography," IEEE Trans. Med. Imag., vol. MI-1, no. 2, pp.113-122, Oct. 1982.

5. E. U. Mumcuo glu, R. Leahy, S. Cherry, and Z. Zhou, "Fast gradient based methods for Bayesian reconstruction of transmission and emission pet images," IEEE Trans Med. Imag., vol. 13, no. 4, pp. 687-701, Dec. 1994.

6. H. Hudson and R. Larkin, "Accelerated image reconstruction using ordered subsets of projection data,"IEEE Trans. Med. Imag., vol. 13, no. 4, pp. 601-609, Dec. 1994

7. Bouman and K. Sauer, "A unified approach to statistical tomography using coordinate descent optimization,” IEEE Trans. Image Process. vol. 5, no. 3, pp. 480-492, Mar. 1996.

8. J. Hsieh, "Accelerated line search for coordinate descent optimization," in Proc. IEEE Nucl. Sci. Symp.Med. Imag. Conf., San Diego, CA, 2006, , vol. 5, pp. 2841- 2844 .

9. J. Fessler and A. Hero, "Penalized maximum-likelihood image reconstruction using space-alternating generalized EM algorithms," IEEE Trans. Image Process., vol. 4, no. 10, pp. 1417-1429, Oct. 1995.

10. H. Erdogan and J. Fessler, "Monotonic algorithms for transmission tomography,” IEEE Trans. Med. Imag., vol. 18, no. 9, pp. 801-814, Sep. 1999.

11. J. Bowsher, M. Smith, J. Peter, and R. Jaszczak, “A comparison of OSEM and ICD for iterative reconstruction of SPECT brain images," J. Nucl. Med., vol. 39, p. 79P, 1998.

12. B. De Man and S. Basu, "Distance-driven projection and backprojection in three-dimensions," Phys. Med. Biol., vol. 49, pp. 2463-2475, 2004.

13. J.Surendiran, Dr.S.V.Saravanan, F.Elizabeth Catherine," GLAUCOMA DETECTION USING FUZZY C- MEAN (FCM)", IJPT| Sep-2016 | Vol. 8 | Issue No.3 | 16149-16163.

14. N. Nithiyanandam, K. Venkatesh, M. Rajesh, Transfer The Levels Of The Monitored Carbon, Nitrogen Gases From The Industries, International Journal of Recent Technology and Engineering, Volume-7 Issue-6S3 April, 2019.

15. Sivanesh Kumar, A., Brittoraj, S., Rajesh, M., Implementation of RFID with internet of things, Journal of Recent Technology and Engineering, Volume-7 Issue-6S3 April, 2019.

16. Rajesh, M., Sairam, R., Big data and health care system using mlearningJournal of Recent Technology and Engineering, Volume-7 Issue-6S3 April, 2019.

17. Rajesh, M., and J. M. Gnanasekar. "Path Observation Based Physical Routing Protocol for Wireless Ad Hoc Networks." Wireless Personal Communications 97.1 (2017): 1267-1289. 